\title{
Low Density Lipoprotein Cholesterol Is Associated With Increased Risk of Cardiovascular Disease in Participants Over 70 Years Old: A Prospective Cohort Study
}

\author{
xin Su \\ Capital Medical University https://orcid.org/0000-0002-7238-6534 \\ Deqiang Zheng \\ Capital Medical University School of Public Health \\ Meiping Wang \\ Capital Medical University School of Public Health \\ Yingting Zuo \\ Capital Medical University School of Public Health \\ Jing Wen \\ Capital Medical University School of Public Health \\ Qi Zhai \\ Capital Medical University School of Public Health \\ Yibo Zhang \\ Capital Medical University School of Public Health \\ Yan He ( $\nabla$ yanhe118@sina.com) \\ Capital Medical University https://orcid.org/0000-0002-6496-8016
}

\section{Original investigation}

Keywords: Elderly, Low-density lipoprotein-cholesterol, Cardiovascular disease, Cohort study

Posted Date: May 11th, 2021

DOI: https://doi.org/10.21203/rs.3.rs-495621/v1

License: () (i) This work is licensed under a Creative Commons Attribution 4.0 International License. Read Full License

Version of Record: A version of this preprint was published at Nutrition, Metabolism and Cardiovascular Diseases on October 1st, 2021. See the published version at https://doi.org/10.1016/j.numecd.2021.10.009. 


\section{Abstract}

\section{Background}

Previous studies, in which the data were collected about half a century ago, suggested that elevated low density lipoprotein cholesterol (LDL-C) is not associated with increased risk of cardiovascular disease (CVD) in patients over 70 years old. However, what is the relationship between LDL-C and CVD risk in a contemporary population aged over 70 years has not been well examined in China.

\section{Methods}

The China Health and Retirement Longitudinal Study (CHARLS) is an ongoing nationally representative study. In this analysis, participants of CHARLS who did not taking statins and did not have heart disease and stroke at 2011 were include and were followed up to 2018 . The outcome of this analysis was occurrence of CVD at follow up, which include heart disease and stroke. Cox regression was used to assess the harmful effect of LDL-C on CVD occurrence. We calculated e-values to quantify the effect of unmeasured confounding on our results.

\section{Results}

Of the 9,631 participants, $15.2 \%(N=1,463)$ were aged over 70 years and $52.5 \%(N=5,060)$ were female. During the 7 years follow-up, 1,437 participants had a first CVD attack. Risk of CVD occurrence increased $8 \%$ with each $10 \mathrm{mg} / \mathrm{mL}$ elevation in LDL-C in whole participants (adjusted HR, 1.08; $95 \% \mathrm{Cl}$, 1.06-1.10) and age groups of $\geq 70$ years, $60-69$ years and $<60$ years. Similar results were observed in subgroup analyses, in which participants were stratified by sex, hypertension, diabetes and chronic kidney disease. According to the restricted cubic spline, we noted a U-shaped relationship between LDL-C and risk of CVD occurrence in group over 70 years old, however, we further found that in the left side of U-shape curve, LDL-C was not associated with occurrence of CVD and its attribution to CVD occurrence was only $2.1 \%$, which indicated that lower level of LDL-C could not increase the risk of CVD occurrence as it was demonstrated by a U-shape association. E-value analysis suggested robustness to unmeasured confounding.

\section{Conclusions}

In contemporary society of China, elevated the level of LDL-C also increased the risk of CVD in participants over 70 years old as in the relatively younger participants. These results should strengthen guideline recommendations for the use of lipid lowering therapies in those elderly.

\section{Introduction}

A large number of epidemiological and clinical studies indicate that arteriosclerotic cardiovascular disease (ASCVD) is currently the leading fatal and disabling disease that endangers human health. The latest report in China shows that the prevalence of cardiovascular disease (CVD) in China is on a continuous rise.[1] It is estimated that there are 330 million people suffering from CVD and its mortality ranks the first. Evidence from genetic studies, animal studies, population-based cohort study and randomized clinical trial, all indicated that LDL-C play a crucial role in CVD occurrence and development. [2,3] At present, most lipid guidelines regard LDL-C as the primary indicator for assessing ASCVD risk and target for intervention. [4-6]

However, several prospective studies have shown that the elevated LDL-C and total cholesterol (TC) appear to be less important risk factors for CVD in people over 70 years old as they were in younger people. [7-9] In some studies, the association of increased cholesterol with clinical events even disappeared in individuals over 70 years. $[10,11]$ However, most of these studies were conducted in the historical cohorts in which the analyzed data were collected about half a century ago. Further, the clinical benefit from LDL-C lowering in older patients remains debated because participants aged 70 years or older were not well represented in individual trials. [12,13] Therefore, guidelines have generally not recommended management of LDL-C with statins in individuals older than 75 years. [6]

In the past few decades, with the substantial changes of living environment, the risk factors for CVD have also changed, [14-16] eg, a study showed that attribution of general obesity for CVD events was very low in1991, however it increased significantly since that time [14] and the prevalence of high LDL-C is also increasing in China. $[17,18]$ Therefore, whether LDL-C is associated with increased risk of CVD occurrence in people over 70 years old in China is deserved to investigated. A most recent study performed in Copenhagen General Population has demonstrated that higher LDL-C is associated with greatly increased absolute risk of myocardial infarction in people aged 70-100 years, which also provided the basis for us to perform this analysis. [19]

Currently, the general population has experienced an increase in life span, [20,21] this will lead to a rapid increase of the absolute number in old people, the projected annual CVD events would increase by $>50 \%$ between 2010 and 2030 in China, mostly in people aged 65-84 years. [22,23] Together, understanding the influence of elevated LDL-C on CVD occurrence in people over 70 years in the current era is important for prevention and treatment of CVD. Therefore, the aim of this study is to assess whether elevated LDL-C were associated with increased risk of CVD occurrence in individuals over 70 years in China.

\section{Methods}

\section{Study design and participants}

The China Health and Retirement Longitudinal Study (CHARLS) is a nationally population-based representative prospective study of Chinese adults aged $\geq 45$ years. The CHARLS is designed to describe the dynamics of retirement and its impact on health, health insurance, and economic well-being. The 
baseline survey was conducted in 2011-2012 among 17,708 participants in 10,257 households were recruited from 150 counties of China's 28 provinces, using the multistage stratified probability-proportional-to-size sampling technique. [24] All participants underwent an assessment using a standardized questionnaire to collect data on socioeconomic status, life style factors and health-related information. All participants were followed up every 2 years after the baseline survey.

The CHARLS data are available for the baseline survey in 2011-2012 (wave 1), the first follow-up survey in 2013-2014 (wave 2), the second follow-up survey in 2015-2016 (wave 3), and the third follow-up survey in 2018-2019 (wave 4). We excluded individuals with preexisting cardiovascular disease (defined as known previous heart disease or stroke), or statin use at baseline. Our final sample size was 9,631 participants. The CHARLS study was approved by the ethical review committee of Peking University and all participants provided written informed consent.

\section{Outcome Ascertainment}

The study outcome was incident CVD. Incident CVD was based on self-reported physician's diagnosis following standardized questions: "Has a doctor ever told you that you had any heart disease [myocardial infarction, coronary heart disease, angina, congestive heart failure, or other heart problems] or "Has a doctor ever told you that you have been diagnosed with a stroke?" Participants who reported heart disease or stroke during the follow-up period were defined as having incident CVD. The date of CVD diagnosis was recorded as being between the date of the last interview and that of the interview reporting an incident CVD.

\section{Determination of lipid profile}

Serum samples were collected after an overnight fast and stored at $-80^{\circ} \mathrm{C}$ until analysis. Biochemical analyses were performed at the Youanmen Center for Clinical Laboratory of Capital Medical University. This laboratory has a regular external quality assessment organized by the Chinese Ministry of Health. [25] Serum total cholesterol (TC), triglycerides (TG), low-density lipoprotein cholesterol (LDL-C), high-density lipoprotein cholesterol (HDL-C) were measured by an enzymatic colorimetric test. Blood glucose was measured by the glucose oxidase method. Glycosylated hemoglobin (HbA1c) was measured by the affinity high performance liquid chromatography (HPLC) method.

\section{Covariates}

At baseline, trained interviewers collected information on social and demographic characteristics and health-related factors using a standard questionnaire, including age, sex (male, female), living residence (rural, urban), marital status (married and other marital status), education level (primary school and below, middle school and above). Self-reported health behaviors included smoking status (never, former, and current smoker), frequency of alcohol consumption in the past year (never, less than once a month, and more than once a month), self-reported physician-diagnosed chronic diseases (diabetes, hypertension, dyslipidemia, and chronic kidney disease), and use of prescribed medications for diabetes, hypertension. Height, weight, and blood pressure were measured by a trained nurse. Body mass index (BMI) was calculated as the weight in kilograms divided by the square of the height in meters.[26]

\section{Definition and calculation}

Hypertension was defined as systolic blood pressure (SBP) $\geq 140 \mathrm{mmHg}$ and / or diastolic blood pressure (DBP) $\geq 90 \mathrm{mmHg}$ or current use of the antihypertensive medication or self-reported history of hypertension. Diabetes was defined as fasting plasma glucose $\geq 126 \mathrm{mg} / \mathrm{dL}$ or current use of antidiabetic medication or self-reported history of diabetes. Chronic kidney disease was defined as estimated glomerular filtration rate $<60 \mathrm{~mL} / \mathrm{min} / 1.73$ $\mathrm{m}^{2}$ or self-reported history of chronic kidney disease. The estimated glomerular filtration rate(eGFR) was calculated using the CKD- EPI equation.[27] Participants attributable risk percent $(P A R P)=\left(I_{t}-I_{0}\right) / I_{t} \times 100 \%, l_{t}$ : incidence of CVD in the participants over 70 years old stratified by LDL-C, $I_{0}$ : incidence of CVD in participants below the median group of LDL-C.

\section{Statistics}

Data were described as means and SDs for normally distributed continuous variables, and as medians and interquartile ranges for nonnormally distributed continuous variables. Frequency with percentage was used to describe categorical variables. Differences in baseline characteristics of the participants by age were evaluated by chi-square, analysis of variance, or Kruskal-Wallis tests, as appropriate.

Cox proportional hazards models were used to assess the association between elevated LDL-C and risk of CVD, in which the demographic characteristics (age, sex, rural residence, married, educational level), behavioral factors (smoking status, drinking status), history of disease and medication (history of comorbidities, history of medication use) and metabolism related status (SBP, DBP, BMI, FPG, HbA1c, TC, TG, HDL-C, eGFR and hs-CRP) were adjusted. These missing data of these covariates were inserted with multiple imputation. We tested the Cox proportional hazard assumption visually for covariates using Shoenfeld residuals and found no violations. In addition, we explored the potential nonlinear associations using 3-knotted restricted cubic spline regression.

Subgroup analyses were conducted in the participants with: sex, hypertension, diabetes, chronic kidney disease. In sensitivity analyses, a simpler E-value technique was used in this study to analysis the potential impact of unmeasured confounding in the association of LDL-C and CVD. The 2-sided significance level was set at $p<0.05$. All statistical analyses were performed using the SPSS 25 software and Stata/SE 15.1 . 


\section{Results}

\section{Baseline characteristics of participants}

Among the 17,708 participants of the CHARLS study, 9,631 were included in this analysis after excluding participants with heart disease, stroke, statin use at baseline, missing data on LDL-C and those who were lost during the 7-year follow-up (Figure 1). Baseline characteristics of the all participants stratified by age are shown in Table 1 . The mean age of the participants was $58.7 \pm 9.8$ years old. The distribution of age for over 70 years were 1,463 (15.2\%), 60-69 years were 2,584 (26.8\%), less than 60 years were 5,584 (58.0\%), respectively. Among the participants, 5,060(52.5\%) were female. The serum level of LDL-C, HDL-C, hs-CRP and SBP are higher in the older group, meanwhile the BMI, TG and eGFR is lower $(P<0.001)$.

\section{Association of LDL-C and occurrence of CVD}

During follow up of 7 years, 1,437 participants experienced incident CVD (heart disease: 1,075 cases; stroke: 362 cases). After adjusting for potential confounders, risk of occurrence of CVD was $8.0 \%$ increased with per $10 \mathrm{mg} / \mathrm{mL}$ increase of LDL-C in whole participants (HR 1.08, 95\% Cl 1.06-1.10) and the association were also observed in all age groups (Figure 2). When considering the individual types of CVD, elevated LDL-C was associated with occurrence of heart disease (HR 1.07, 95\% Cl 1.06-1.09) and stroke (HR 1.10,95\% Cl 1.07-1.13) in the all participants and in all age groups (Figure 2).

In order to eliminate the influence of some factors on the association between LDL-C and CVD, we stratified the participants into subgroups by the potential influence factors. Similar results were demonstrated in different subgroups (Table 2).

\section{U-shape association between LDL-C for risk of CVD occurrence}

To examine whether there is an optimal range of LDL-C, in which the risk of CVD occurrence is the lowest, a multivariable regression analysis with restricted cubic spline were conducted. We found a U-shape association between the level of LDL-C and risk of CVD occurrence in group $\geq 70$ years. The level of LDL-C associated with the lowest risk of CVD in multivariable adjusted analyses was $2.8 \mathrm{mmol} / \mathrm{L}(110 \mathrm{mg} / \mathrm{dL})$. Whereas, they were both linear and positive association in the groups of aged 60-69 years and $<60$ years (Figure 3 ). Similar curves were observed for the associations of the LDL-C with incident heart disease and stroke, respectively, in the group $\geq 70$ years. (eFigure 1 and 2 in the Supplement).

\section{Possible reasons for the U-shape association between levels of LDL-C and CVD occurrence in the participants over 70 years old}

Since we had demonstrated a U-shape association between levels of LDL-C and CVD occurrence in participants over 70 years old, to critically assess whether the lower level of LDL-C increased risk of CVD occurrence, we divided the participants over 70 years old into 2 groups by the LDL-C level(110mg/dL), which corresponding to the lowest CVD risk. The baseline characteristics of participants with LDL-C level $<110 \mathrm{mg} / \mathrm{dL}$ or $\geq 110 \mathrm{mg} / \mathrm{dL}$ are shown in eTable1. After adjusting for potential confounders, in the group of LDL-C $<110 \mathrm{mg} / \mathrm{dL}$, occurrence of CVD was not associated with LDL-C (HR $1.02,95 \% \mathrm{Cl} 0.86-1.22$ ) (Table3), but was associated with history of dyslipidemia, drinking and increased DBP. However, in the group of LDL-C $\geq 110 \mathrm{mg} / \mathrm{dL}$, elevated LDL-C was associated with occurrence of CVD (HR 1.13, 95\% Cl 1.08-1.19) (Table3). To further assess the contribution of LDL-C to the occurrence of CVD, we calculated the PARP in the participants over 70 years old (eTable 2). The PARP was $2.1 \%$ for the group of LDL-C < $110 \mathrm{mg} / \mathrm{dL}$ and $14.5 \%$ for the group of LDL-C $\geq 110 \mathrm{mg} / \mathrm{dL}$.

\section{E-value assessment}

Sensitivity analysis was conducted to assess the robustness of the association to unmeasured confounding. The E-value for the observed point-estimate of LDL-C and CVD for all participants (HR 1.08[95\% Cl 1.06, 1.10]) and over 70 years old (HR $1.08[95 \% \mathrm{Cl} 1.05,1.12])$ was $1.37,1.38$, respectively. The observed increased incidence of CVD with an increase of LDL-C in all participants and over 70 years old could be explained away by unmeasured confounding that was associated with LDL-C and CVD by an HR of 1.37 and 1.38, above and beyond the measured confounders, but not by weaker confounding. The corresponding $\mathrm{Cl}$ could be moved to include the null by an unmeasured confounder that was associated with both LDL-C and CVD by an HR of at least $1.32,1.25$, respectively.

\section{Discussion}

In this prospective study, we demonstrated that elevated LDL-C was associated with increased risk of CVD occurrence in participants over 70 years. This association was further confirmed by the subgroup analyses conducted in different sex and participant with or without hypertension, diabetes and chronic kidney disease, respectively. This is the first study to systematically evaluate the effects of LDL-C on CVD occurrence in participants with the age over 70 years in China.

Previous studies have suggested that the association of elevated total cholesterol and LDL cholesterol levels with increased risk of CVD decreases substantially with increasing age, [7-9] or even disappeared in individuals over 70 years. [10,11] For example, G David Batty [7] reported from the Whitehall study (enrolling participants in 1967-70) that the associations of total cholesterol with coronary heart disease and stroke diminished in strength with increasing duration of follow-up. Harlan M [11] reported that raised total cholesterol or low HDL-C was not important risk factors for coronary heart disease mortality and morbidity and all-cause mortality in people over 70 years. By contrast, in our study, the risk of CVD increased with higher LDL cholesterol levels in all age groups even if individuals over 70 years. This result is similar to a most recent study from the Copenhagen General Population Study, which showed people aged 70-100 years with raised LDL cholesterol had the greatly increased absolute risk of myocardial infarction and atherosclerotic cardiovascular disease.[19] 
Most previous studies investigating the association of elevated cholesterol with risk of CVD were conducted in the historical cohorts in which participants and analyzed data were collected about half a century ago. Since then, with the substantial changes of living environment, the general population has experienced an increase of life span. [20,21] Therefore, the life span for the subjects over 70 years old had also increased, it means that those elderly people would undergo an even longer period of the unfavorable effect of LDL-C on CVD occurrence. This might account for the positive association between LDL-C and occurrence of CVD in the participants over 70 years in this study.

Several randomized controlled trials showed further lowering of LDL-C beyond the lowest current targets (70mg/dL) would further reduce cardiovascular risk. [28-30] However, in this study, use multivariable regression with restricted cubic spline, we found the association between levels of LDL-C and risk of CVD was U shaped in group over 70 years old. Whether lower levels of LDL-C can really increase risk of CVD occurrence? We denied this conclusion by further analysis conducted in the participants over 70 years old: (1) In the group of LDL-C $<110 \mathrm{mg} / \mathrm{dL}$, occurrence of CVD was not associated with LDL-C, but was associated with history of dyslipidemia, drinking and increased DBP. However, in the group of LDL-C $\geq 110 \mathrm{mg} / \mathrm{dL}$, elevated LDL-C was associated with occurrence of CVD. (2) The attribution of LDL-C to CVD occurrence was lower in the participants with LDL-C $<110 \mathrm{mg} / \mathrm{dL}$ (2.1\%) vs those with LDL$C \geq 110 \mathrm{mg} / \mathrm{dL}$ (14.5\%). These results all indicated that when LDL-C was lower than $110 \mathrm{mg} / \mathrm{dL}$, lower levels of LDL-C did not increase the risk of CVD occurrence.

Several previous studies had provided some clues for understanding the mechanisms underlying this negative association between LDL-C and CVD occurrence. First, this might be a reverse causation. In our study the mortality rate is higher in participants with $L D L-C<110 \mathrm{mg} / \mathrm{dL}$, previous study found debilitation and illness have been hypothesized to cause a decrease in levels of cholesterol. [31,32] Therefore, the decline of LDL-C may be caused by the frailty of the elderly, this hypothesis could be supported by the lower level of glucose $(107.3 \mathrm{mg} / \mathrm{dL}$ vs $112.3 \mathrm{mg} / \mathrm{dL}$ ) and other lipids profile (TC: 163.9 $\mathrm{mg} / \mathrm{dL}$ vs $216.6 \mathrm{mg} / \mathrm{dL}, \mathrm{TG}: 86.7 \mathrm{mg} / \mathrm{dL}$ vs $103.5 \mathrm{mg} / \mathrm{dL}$ ) in this group of participants versus participants with the LDL-C $\geq 110 \mathrm{mg} / \mathrm{dL}$. Hence, we thought a high mortality rate may be a contributing "cause" of LDL-C decreased. Second, despite extensive adjustment, but whether exist residual confounding is unclear. Taken together, these data suggest that statin therapy in individuals over 70 years with high cholesterol could be appropriate, this view supports the opinion of RCT. [33,34] But, what is the appropriate level of LDL-C in subjects older than 70 years deserves careful investigation. The ongoing randomized controlled trial in 18,000 patients older than 70 years and without cardiovascular disease may clarify the efficacy of statin versus placebo to reduce major vascular events in an older primary prevention population.

We used the E-value sensitivity analysis to quantify the potential implications of unmeasured confounders and found that an unmeasured or unknown confounder was unlikely to explain the effect on LDL-C and CVD (relative risk exceeding 1.37) than these known risk factors.

The strengths of our study include, Firstly, our study is a well-designed large-scale prospective study, the long follow-up period, and the subjects came from different regions of China, therefor their locations were generally representative. Secondly, we adjusted for several confounders for association between LDL-C and CVD, and subgroup analysis were performed in participants with or without some risks of CVD, such as hypertension, diabetes, chronic kidney disease. Finally, a novelty in this population-based study was using the recently introduced E-value as an alternative to sensitivity analysis.

This study also has some limitations. First, self-reported physician-diagnosed incident CVD, could leading to potential misclassification of CVD cases. Second, we only had information on blood lipid at baseline and cannot rule out that the results might been influenced by longitudinal changes during follow-up. Third, we could not deal with the question of causality because the observational design of the study.

\section{Conclusion}

This study found that elevated LDL-C was associated with increased risk of CVD in participants over 70 years old. These data are of importance for primary prevention strategies and guidelines aimed at managing and reducing CVD in the growing older population.

\section{Abbreviations}

LDL-C: low density lipoprotein cholesterol; CVD: Cardiovascular disease; CHARLS: China Health and Retirement Longitudinal Study; ASCVD: Arteriosclerotic cardiovascular disease; TC: Total cholesterol; TG: Triglycerides; HDL-C: High-density lipoprotein cholesterol; BMI: Body mass index; SBP: Systolic blood pressure; DBP, Diastolic blood pressure; FPG, fasting plasma glucose; HbA1c: Hemoglobin A1c; hs-CRP: High-sensitivity C-reactive protein; eGFR: Estimated glomerular filtration rate; CKD: Chronic kidney disease; HR: Hazard ratio; PARP: Participants attributable risk percent.

\section{Declarations}

Ethics approval and consent to participate: Data of this study was from the China Health and Retirement Longitudinal Study (CHARLS). The CHARLS study was approved by the ethical review committee of Peking University and all participants provided written informed consent

\section{Consent for publication: Not applicable}

Availability of data and materials: The data that support the findings of this study are available from The China Health and Retirement Longitudinal Study, but restrictions apply to the availability of these data, which were used under license for the current study, and so are not publicly available. Data are however available from the authors upon reasonable request and with permission of The China Health and Retirement Longitudinal Study

Competing interests: The authors declare that they have no competing interests 
Funding: This research was funded by the National Natural Science Foundation of China, grant number 82073648

Authors' contributions: XS and $\mathrm{YH}$, was responsible for the study concept and design. XS, analyzed data and wrote the manuscript. YH, obtained the funding. XS, DQZ, MPW, YTZ, JW, QZ, YBZ, performed statistical analyses. XS, YH, contributed to data interpretation and the editing of the manuscript. All authors read and approved the final manuscript.

Acknowledgements: This research used data from the China Health and Retirement Longitudinal Study (CHARLS). This Study was developed by a team of researchers based at Peking University. We sincerely thank those who participated in data collection and management.

\section{References}

1. 1. Zhao D, Liu J, Wang M, et al. Epidemiology of cardiovascular disease in China: current features and implications. Nat Rev Cardiol.2019 Apr;16(4):203-212.

2. 2. Ross R. Atherosclerosis-An Inflammatory Disease. N Engl J Med 1999; 340(2):115-126.

3. 3. Borén J, Chapman MJ, Krauss RM, et al. Low-density lipoproteins cause atherosclerotic cardiovascular disease: pathophysiological, genetic, and therapeutic insights: a consensus statement from the European Atherosclerosis Society Consensus Panel. Eur Heart J. 2020 ;41(24):2313-2330.

4. 4. Mach F, Baigent C, Catapano AL, et al. 2019 ESC/EAS Guidelines for the management of dyslipidaemias: lipid modification to reduce cardiovascular risk. Eur Heart J. 2020; 41(1): 111-188.

5. 5. Grundy SM, Stone NJ, Bailey AL, et al. 2018 AHA/ACC/AACVPR/AAPA/ABC/

6. ACPM/ADA/AGS/APhA/ASPC/NLA/PCNA guideline on the management of blood cholesterol: executive summary-a report of the American College of Cardiology/ American Heart Association Task Force on Clinical Practice Guidelines. J Am Coll Cardio/ 2019; 73 : 3168 - 209.

7. 6. Kinoshita M, Yokote K, Arai H, et al. Japan Atherosclerosis Society (JAS) Guidelines for Prevention of Atherosclerotic Cardiovascular Diseases 2017. J Atheroscler Thromb 2018; 25(9):846-984.

8. 7. Batty GD, Shipley M, Smith GD, et al. Long term risk factors for coronary heart disease and stroke: influence of duration of follow-up over four decades of mortality surveillance. Eur J Prev Cardiol. 2015; 22: 1139-1145.

9. 8. Simons LA, Simons J, Friedlander Y, et al. Cholesterol and other lipids predict coronary heart disease and ischemic stroke in the elderly, but only in those below 70 years. Atherosclerosis. 2001; 159: 201-208.

10. 9. Clarke R, Lewington S, Youngman L, et al. Underestimation of the importance of blood pressure and cholesterol for coronary heart disease mortality in old age. Eur Heart J. 2002; 23: 286-293.

11. 10. Iversen A, Jensen JS, Scharling $\mathrm{H}$, et al. Hypercholesterolemia and risk of coronary heart disease in the elderly: impact of age: the Copenhagen City Heart Study. Eur J Intern Med 2009; 20: 139 - 44.

12. 11. Krumholz HM, Seeman TE, Merrill SS, et al. Lack of association between cholesterol and coronary heart disease mortality and morbidity and allcause mortality in persons older than 70 years. JAMA 1994; 272: 1335-40.

13. 12. Chou R, Dana T, Blazina I, et al. Statins for prevention of cardiovascular disease in adults: evidence report and systematic review for the US preventive services task force. JAMA 2016; 316: 2008-24.

14. 13. Mortensen MB, Falk E. Primary prevention with statins in the elderly. J Am Coll Cardiol 2018; 71: 85-94.

15. 14. Li Y, Wang DD, Ley SH, et al. Potential Impact of Time Trend of Life-Style Factors on Cardiovascular Disease Burden in China. J Am Coll Cardiol 2016;68(8):818- 33 .

16. 15. Global burden of metabolic risk factors for chronic diseases collaboration. Cardiovascular disease, chronic kidney disease, and diabetes mortality burden of cardiometabolic risk factors from 1980 to 2010: a comparative risk assessment. Lancet Diabetes Endocrinol. $2014 ;$ 2: 634-647.

17. 16. Lim SS, Vos T, Flaxman AD, et al. A comparative risk assessment of burden of disease and injury attributable to 67 risk factors and risk factor clusters in 21 regions, 1990-2010: a systematic analysis for the Global Burden of Disease Study 2010. Lancet. 2012; 380(9859):2224-60.

18. 17. Li JZ, Wang S, Dong J, et al. Present status of serum lipid levels in Beijing professional populations and its trend of changes over 15 years-a collaborative study of seven research and clinical laboratories in Beijing. Clin Chim Acta 2005; 352(1-2): $199-207$.

19. 18. Yang W, Xiao J, Yang Z, et al. Serum lipids and lipoproteins in Chinese men and women. Circulation 2012; 125: $2212-21$.

20. 19. Mortensen MB, Nordestgaard BG. Elevated LDL cholesterol and increased risk of myocardial infarction and atherosclerotic cardiovascular disease in individuals aged 70-100 years: a contemporary primary prevention cohort. Lancet. 2020; 396(10263): 1644-1652.

21. 20. Zhou M, Wang H, Zhu J, et al. Cause-specific mortality for 240 causes in China during 1990-2013: a systematic subnational analysis for the Global Burden of Disease Study 2013. Lancet 2016; 387(10015): 251-272.

22. 21. GBD 2013 DALYs and HALE Collaborators. Global, regional, and national disability-adjusted life years (DAL Ys) for 306 diseases and injuries and healthy life expectancy (HALE) for 188 countries, 1990-2013: quantifying the epidemiological transition. Lancet 2015; 386: 2145-2191.

23. 22. Moran A, Gu D, Zhao D, et al. Future cardiovascular disease in China: markov model and risk factor scenario projections from the coronary heart disease policy model-China. Circ Cardiovasc Qual Outcomes 2010; 3(3): 243-252.

24. 23. Moran A. Zhao D, Gu D, et al. The future impact of population growth and aging on coronary heart disease in China: projections from the Coronary Heart Disease Policy Model-China. BMC Public Health 2008; 8: 394. 
25. 24. Zhao Y, Hu Y, Smith JP, et al. Cohort profile: the China Health and Retirement Longitudinal Study (CHARLS). Int J Epidemiol. 2014;43(1):61-68.

26. 25. Chen X, Crimmins E, Hu PP, et al. Venous Blood-Based Biomarkers in the China Health and Retirement Longitudinal Study: Rationale, Design, and Results From the 2015 Wave. Am J Epidemiol. 2019;188(11):1871-1877.

27. 26. Zhao Yaohui, Yisong Hu, James P Smith, et al. Cohort Profile: The China Health and Retirement Longitudinal Study (CHARLS). International Journal of Epidemiology, 2014; 43(1): 61-68.

28. 27. Levey AS, Stevens LA, Schmid CH, et al. A new equation to estimate glomerular filtration rate. Ann Intern Med 2009;150(9): 604 - 12.

29. 28. Cannon CP, Blazing MA, Giugliano RP, et al. IMPROVE-IT Investigators. Ezetimibe added to statin therapy after acute coronary syndromes. N EngI J Med. 2015; 372(25): 2387-2397.

30. 29. Sabatine MS, Giugliano RP, Keech AC, et al. FOURIER Steering Committee and Investigators. Evolocumab and clinical outcomes in patients with cardiovascular disease. N Engl J Med. 2017; 376(18): 1713-1722.

31. 30. Bowman L, Hopewell JC, Chen F, et al. HPS3/TIMI55-REVEAL Collaborative Group. Effects of anacetrapib in patients with atherosclerotic vascular disease. N Engl J Med. 2017;377(13):1217-1227.

32. 31. Jacobs D, Blackburn H, Higgins M, et al. Report of the Conference on Low Blood Cholesterol: mortality associations. Circulation 1992;86: 104660.

33. 32. Ranieri P, Rozzini R, Franzoni S, et al. Serum cholesterol levels as a measure of frailty in elderly patients. Exp Aging Res 1998 ; 24 : 169 - 79.

34. 33. Ouchi Y, Sasaki J, Arai H, et al. Ezetimibe Lipid-Lowering Trial on Prevention of Atherosclerotic Cardiovascular Disease in 75 or Older (EWTOPIA 75): A Randomized, Controlled Trial. Circulation. 2019; 140(12): 992-1003.

35. 34. Bach RG, Cannon CP, Giugliano RP, et al. Effect of Simvastatin-Ezetimibe Compared With Simvastatin Monotherapy After Acute Coronary Syndrome Among Patients 75 Years or Older: A Secondary Analysis of a Randomized Clinical Trial. JAMA Cardiol. 2019; 4(9): 846-854.

\section{Tables}

Table 1 Baseline characteristics of participants 


\begin{tabular}{|c|c|c|c|c|c|}
\hline & $\begin{array}{l}\text { All Participants } \\
(n=9631)\end{array}$ & $\begin{array}{l}\text { Aged } \geq 70 \text { years } \\
(n=1463)\end{array}$ & $\begin{array}{l}\text { Aged 60-69 years } \\
(n=2584)\end{array}$ & $\begin{array}{l}\text { Aged }<60 \text { years } \\
(n=5584)\end{array}$ & $P$-value \\
\hline Age(years) & $58.7(9.8)$ & $75.5(4.8)$ & $63.8(2.8)$ & $51.9(4.8)$ & $<0.001$ \\
\hline Female $(n, \%)$ & $5060(52.5)$ & $698(47.7)$ & $1310(50.7)$ & $3088(55.3)$ & $<0.001$ \\
\hline Rural residence & $6260(65.0)$ & $1001(68.4)$ & 1735(67.1) & $3524(63.1)$ & $<0.001$ \\
\hline Married & 7994(83.0) & $946(64.6)$ & 2168(83.9) & $4880(87.4)$ & $<0.001$ \\
\hline Educational level & & & & & $<0.001$ \\
\hline Primary school and below & $6655(69.1)$ & 1303(89.1) & 2086(80.7) & $3266(58.5)$ & \\
\hline Middle school and above & 2976(30.9) & $160(10.9)$ & 498(19.3) & 2318(41.5) & \\
\hline Smoking status & & & & & $<0.001$ \\
\hline Never & $5838(60.6)$ & $833(56.9)$ & 1464(56.7) & $3545(63.5)$ & \\
\hline Former & 753(7.8) & 183(12.5) & $244(9.4)$ & $324(5.8)$ & \\
\hline Current & $3040(31.6)$ & $447(30.6)$ & 876(33.9) & 1715(30.7) & \\
\hline Drinking status & & & & & $<0.001$ \\
\hline Never & 7453(77.4) & $1126(76.0)$ & 1916(74.2) & $4411(79.0)$ & \\
\hline Former & $910(9.4)$ & 169(11.5) & $331(12.8)$ & $410(7.3)$ & \\
\hline Current & 1268(13.2) & 168(11.5) & $337(13.0)$ & 763(13.7) & \\
\hline \multicolumn{6}{|l|}{ History of comorbidities } \\
\hline Diabetes & $372(3.9)$ & $56(3.8)$ & $120(4.6)$ & 194(3.5) & 0.03 \\
\hline Hypertension & 1920(19.9) & $403(27.6)$ & $651(25.2)$ & $859(15.4)$ & $<0.001$ \\
\hline Dyslipidemia & $377(3.9)$ & $46(3.1)$ & 106(4.1) & 219(3.9) & 0.24 \\
\hline Chronic kidney disease & $537(5.6)$ & $72(4.9)$ & $158(6.1)$ & $304(5.4)$ & 0.25 \\
\hline \multicolumn{6}{|l|}{ History of medication use } \\
\hline Antidiabetic medications & $233(2.4)$ & $32(2.2)$ & 83(3.2) & 118(2.1) & 0.01 \\
\hline Antihypertensive medications & $1570(16.3)$ & $340(23.2)$ & $531(20.6)$ & $699(12.5)$ & $<0.001$ \\
\hline $\mathrm{BMI}\left(\mathrm{km} / \mathrm{m}^{2}\right)$ & $23.2(3.5)$ & $21.7(3.6)$ & $23.0(3.5)$ & 23.7(3.4) & $<0.001$ \\
\hline $\mathrm{SBP}(\mathrm{mmHg})$ & $111.9(17.7)$ & 118.2(18.9) & 113.6(18.0) & 109.3(16.6) & $<0.001$ \\
\hline $\mathrm{DBP}(\mathrm{mmHg})$ & $74.4(9.2)$ & $73.4(9.2)$ & 74.3(8.8) & $74.7(9.4)$ & $<0.001$ \\
\hline $\mathrm{FPG}(\mathrm{mg} / \mathrm{dL})$ & 108.8(34.1) & 110.3(34.6) & $111.0(36.7)$ & 107.4(32.6) & $<0.001$ \\
\hline $\mathrm{HbA1c}(\%)$ & $5.2(0.8)$ & $5.2(0.8)$ & $5.3(0.8)$ & $5.2(0.8)$ & $<0.001$ \\
\hline $\mathrm{TC}(\mathrm{mg} / \mathrm{dL})$ & 192.6(37.9) & 193.4(39.6) & 194.5(37.8) & 191.4(37.5) & 0.002 \\
\hline $\mathrm{TG}(\mathrm{mg} / \mathrm{dL})$ & 103.5(74.3-151.3) & $96.5(70.8-138.1)$ & 104.4(74.3-151.3) & 105.3(74.3-155.8) & $<0.001$ \\
\hline $\mathrm{HDL}-\mathrm{C}(\mathrm{mg} / \mathrm{dL})$ & $51.8(15.0)$ & $54.1(15.5)$ & $52.1(15.5)$ & $51.0(14.5)$ & $<0.001$ \\
\hline LDL-C (mg/dL) & 115.7(34.6) & $117.5(34.8)$ & 117.3(35.4) & 114.5(34.1) & $<0.001$ \\
\hline $\mathrm{hs}-\mathrm{CRP}(\mathrm{mg} / \mathrm{L})$ & $1.0(0.53-2.09)$ & $1.3(0.7-3.0)$ & $1.1(0.6-2.2)$ & $0.9(0.5-1.9)$ & $<0.001$ \\
\hline $\operatorname{eGFR}\left(\mathrm{mL} / \mathrm{min} / 1.73 \mathrm{~m}^{2}\right)$ & $92.7(15.0)$ & 78.2(14.8) & $88.7(12.5)$ & $98.4(12.7)$ & $<0.001$ \\
\hline
\end{tabular}

Data were expressed as mean (SD) or median (IQR 25\%-75\%) for continuous variables and as number (percentage) for categorical variables. $P$ from ANOVA, Kruskal-Wallis test or chi- square test.

BMI, body mass index; SBP, systolic blood pressure; DBP, diastolic blood pressure; FPG, fasting plasma glucose; HbA1c, glycated hemoglobin A1c; TC, total cholesterol; TG, triglyceride; HDL- C, high- density lipoprotein cholesterol; LDL- C, low- density lipoprotein cholesterol; hs-CRP, high-sensitivity Creactive protein; eGFR, estimated glomerular filtration rate.

Table 2 Association of LDL cholesterol with occurrence of CVD, stratified by different factors 


\begin{tabular}{|c|c|c|c|c|c|c|c|c|}
\hline \multirow[t]{2}{*}{ Factor } & \multicolumn{2}{|c|}{ ALL Participants } & \multicolumn{2}{|c|}{ Aged $\geq 70$ years } & \multicolumn{2}{|c|}{ Aged $60-69$ years } & \multicolumn{2}{|c|}{ Aged $<60$ years } \\
\hline & $\begin{array}{l}\text { Events(n) / } \\
\text { Participants } \\
\text { (N) }\end{array}$ & $\mathrm{HR}(95 \% \mathrm{Cl})$ & $\begin{array}{l}\text { Events(n) / } \\
\text { Participants } \\
\text { (N) }\end{array}$ & $\mathrm{HR}(95 \% \mathrm{Cl})$ & $\begin{array}{l}\text { Events(n) / } \\
\text { Participants } \\
\text { (N) }\end{array}$ & $\mathrm{HR}(95 \% \mathrm{Cl})$ & $\begin{array}{l}\text { Events(n) / } \\
\text { Participants } \\
\text { (N) }\end{array}$ & $\mathrm{HR}(95 \% \mathrm{Cl})$ \\
\hline \multicolumn{9}{|l|}{ Sex } \\
\hline Male & $662 / 4571$ & $1.08(1.05,1.10)$ & $134 / 765$ & $1.07(1.02,1.13)$ & $207 / 1310$ & $1.01(0.92,1.10)$ & $281 / 2496$ & $1.07(1.03,1.10)$ \\
\hline Female & $815 / 5060$ & $1.08(1.06,1.10)$ & $139 / 698$ & $1.07(1.03,1.13)$ & $260 / 1274$ & $1.07(1.03,1.10)$ & $416 / 3088$ & $1.10(1.07,1.12)$ \\
\hline \multicolumn{9}{|c|}{ Hypertension } \\
\hline No & $899 / 7068$ & $1.10(1.08,1.12)$ & $162 / 929$ & $1.09(1.05,1.14)$ & $275 / 1766$ & $1.09(1.06,1.12)$ & $457 / 4366$ & $1.11(1.08,1.14)$ \\
\hline Yes & $538 / 2563$ & $1.05(1.02,1.07)$ & $111 / 534$ & $1.06(1.00,1.12)$ & $192 / 818$ & $1.05(1.01,1.09)$ & $240 / 1218$ & $1.05(1.01,1.08)$ \\
\hline \multicolumn{9}{|c|}{ Diabetes } \\
\hline No & $1181 / 8220$ & $1.08(1.06,1.10)$ & $225 / 1207$ & $1.07(1.03,1.12)$ & $372 / 2163$ & $1.06(1.04,1.09)$ & $583 / 4851$ & $1.08(1.06,1.11)$ \\
\hline Yes & $256 / 1411$ & $1.08(1.05,1.11)$ & $48 / 256$ & $1.09(1.00,1.19)$ & $95 / 421$ & $1.09(1.04,1.14)$ & $114 / 733$ & $1.09(1.04,1.14)$ \\
\hline \multicolumn{9}{|l|}{ CKD } \\
\hline No & $1296 / 8829$ & $1.08(1.07,1.10)$ & $233 / 1222$ & $1.09(1.06,1.13)$ & $419 / 2361$ & $1.07(1.04,1.10)$ & $645 / 5246$ & $1.08(1.06,1.11)$ \\
\hline Yes & $141 / 802$ & $1.07(1.02,1.12)$ & $40 / 241$ & $1.08(0.80,1.46)$ & $48 / 223$ & $1.10(1.02,1.18)$ & $52 / 338$ & $1.12(1.05,1.20)$ \\
\hline
\end{tabular}

CKD, chronic kidney disease. HR $(95 \% \mathrm{Cl})$, hazard ratios with 95\% confidence interval. Models were adjusted for age, sex, residence, marital status, educational level, smoking status, drinking status, history of diabetes, hypertension, dyslipidemia, and chronic kidney disease; and use hypertension medications, diabetes medications, systolic blood pressure, diastolic blood pressure, fasting plasma glucose, glycated hemoglobin A1c, total cholesterol, triglyceride, high- density lipoprotein cholesterol, low- density lipoprotein cholesterol, hypersensitive C-reactive protein, estimated glomerular filtration rate, body-mass index.

Table 3 Risk factors for occurrence of CVD in participants over 70 years old stratified by LDL-C

\begin{tabular}{|c|c|c|c|c|c|c|}
\hline \multirow[t]{3}{*}{ Risk factors } & \multicolumn{2}{|l|}{ CVD } & \multicolumn{2}{|l|}{ Heart disease } & \multicolumn{2}{|l|}{ Stroke } \\
\hline & Events(n) / & $\mathrm{HR}(95 \% \mathrm{Cl})$ & Events(n) / & $\mathrm{HR}(95 \% \mathrm{Cl})$ & Events(n) / & $\mathrm{HR}(95 \% \mathrm{Cl})$ \\
\hline & Participants (N) & & Participants (N) & & Participants (N) & \\
\hline LDL-C $<110 \mathrm{mg} / \mathrm{dL}$ & $102 / 643$ & & $70 / 643$ & & $32 / 643$ & \\
\hline History of dyslipidemia & $4 / 15$ & $2.47(1.01,6.08)$ & $3 / 15$ & $2.82(1.02,7.76)$ & $1 / 15$ & NA \\
\hline Drinking & $17 / 95$ & $1.39(1.11,1.75)$ & $14 / 95$ & $1.71(1.28,2.29)$ & $3 / 95$ & NA \\
\hline DBP & & $1.03(1.01,1.05)$ & & NA & & $1.04(1.01,1.08)$ \\
\hline FPG & & NA & & NA & & $1.01(1.01,1.02)$ \\
\hline LDL-C & & $1.02(0.86,1.22)$ & & $1.11(0.88,1.41)$ & & $0.88(0.67,1.14)$ \\
\hline $\mathrm{LDL}-\mathrm{C} \geq 110 \mathrm{mg} / \mathrm{dL}$ & $171 / 820$ & & $117 / 820$ & & $54 / 820$ & \\
\hline History of hypertension & $59 / 237$ & $1.45(1.06,1.98)$ & $39 / 237$ & & $20 / 237$ & NA \\
\hline History of dyslipidemia & $12 / 31$ & NA & $11 / 31$ & $2.45(1.32,4.56)$ & $1 / 31$ & NA \\
\hline DBP & & NA & & NA & & $1.02(1.00,1.03)$ \\
\hline Drinking & $21 / 73$ & $1.27(1.03,1.57)$ & $11 / 73$ & NA & $10 / 73$ & $1.78(1.27,2.48)$ \\
\hline LDL-C & & $1.13(1.08,1.19)$ & & $1.09(1.02,1.16)$ & & $1.21(1.12,1.31)$ \\
\hline
\end{tabular}

CVD, cardiovascular diseases; DBP, diastolic blood pressure; FPG, fasting plasma glucose; LDL- C, low- density lipoprotein cholesterol; HR (95\%CI), hazard ratios with 95\% confidence interval. DBP, FPG, LDL-C as continuous variables, history of dyslipidemia, history of dyslipidemia, drinking, as categories variables were entered into the Cox proportional hazards models. NA, not a risk factor.

\section{Figures}




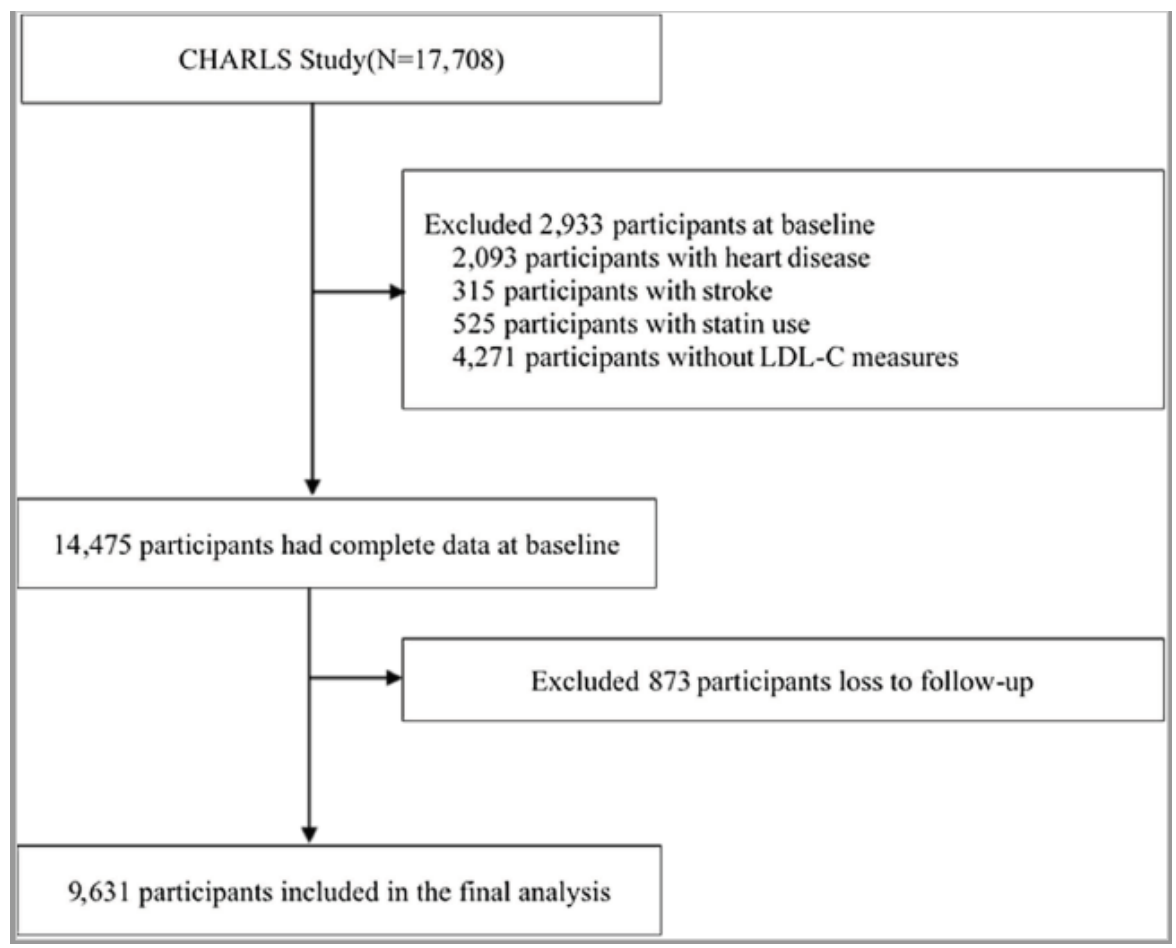

\section{Figure 1}

Flowchart of participants from the CHARLS Study

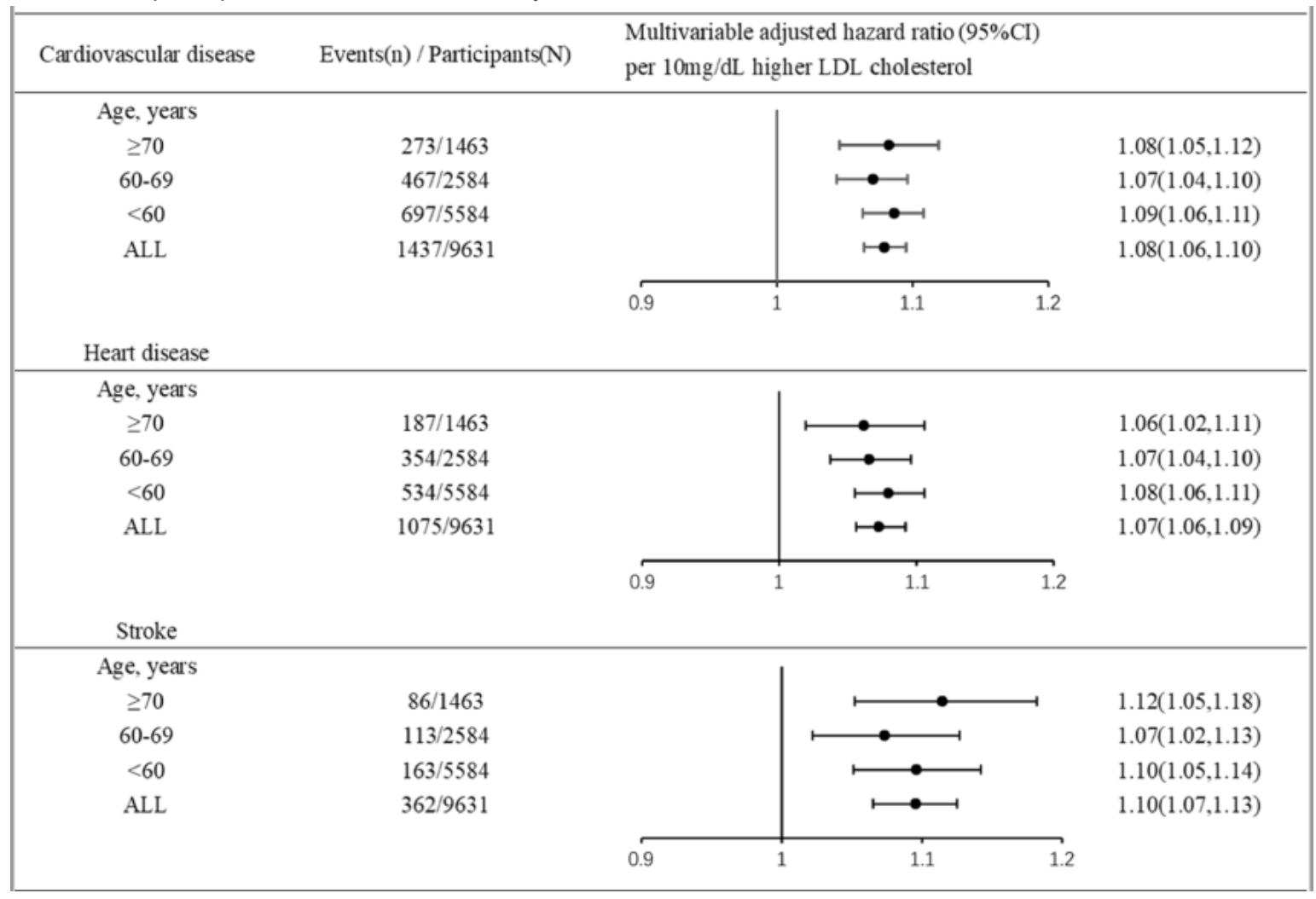

\section{Figure 2}

Association of LDL-C with occurrence of CVD, stratified by age groups Multivariable analyses were adjusted for age, sex, residence, marital status, educational level, smoking status, drinking status, history of diabetes, hypertension, dyslipidemia, and chronic kidney disease; and use hypertension medications, diabetes medications, systolic blood pressure, diastolic blood pressure, fasting plasma glucose, glycated hemoglobin A1c, total cholesterol, triglyceride, high- density lipoprotein cholesterol, low- density lipoprotein cholesterol, hypersensitive C-reactive protein, estimated glomerular filtration rate, body-mass index. 

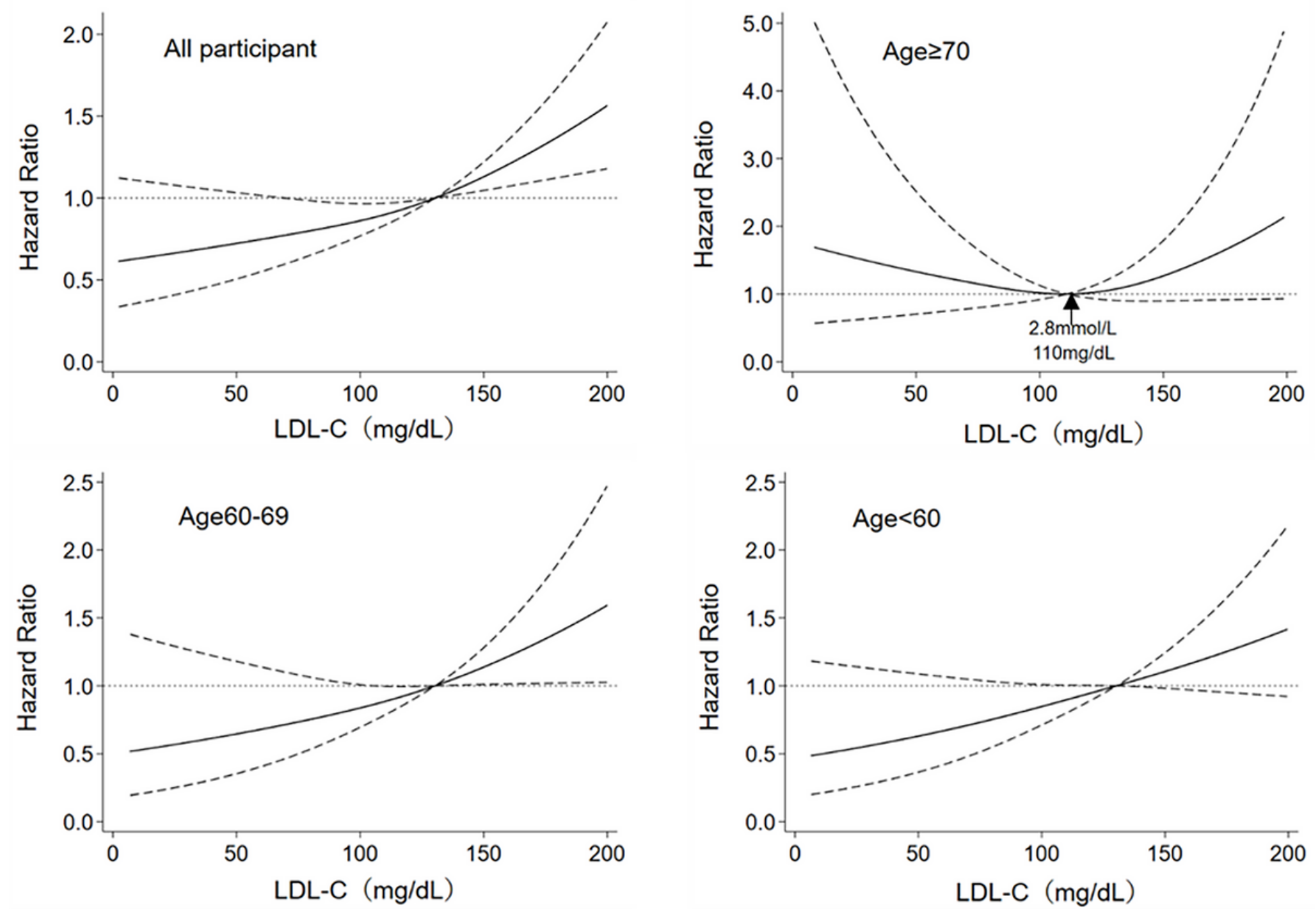

Figure 3

Multivariable adjusted hazard ratios for CVD according to levels of LDL-C on a continuous scale. Solid lines are multivariable adjusted hazard ratios, with dashed lines showing $95 \%$ confidence intervals derived from restricted cubic spline regressions with three knots. Arrows indicate the concentration of LDL-C with the lowest risk of CVD.

\section{Supplementary Files}

This is a list of supplementary files associated with this preprint. Click to download.
- eTable1.xls
- eTable2.xls
- efigure1.tif
- efigure2.tif 